\title{
Ethnobotanical study of traditional herbal plants used by local people of Seshachalam Biosphere Reserve in Eastern Ghats, India
}

\author{
ARAVEETI MADHUSUDHANA REDDY ${ }^{1 *}$, MADHA VENKATA SURESH BABU ${ }^{2}$, RAMACHANDRA \\ RAGHAVENDRA RAO ${ }^{3}$
}

${ }^{1}$ Department of Botany

Yogi Vemana University, Vemanapuram

516005 Kadapa, A.P.

India

${ }^{2}$ Department of Botany

Govt. Degree College, Rajempeta

516115 Kadapa, A.P.

India

${ }^{3}$ INSA Honorary Scientist

Bangalore 560038

India

* corresponding author: phone: 994977 9166, fax: + 91-(0)8562-225419, e-mail: grassced@yahoo.com

\section{Summary}

Introduction: Ethnobotany is the study of medicinal plants used by local people, with particular importance of old-styled tribal beliefs and information. Ethnobotanical studies focus on ethnic knowledge of Adivasi people and development of data bases on ethnic knowledge but also focuses on preservation and regeneration of traditional beliefs and maintenance of traditional knowledge.

Objective: The aim of present study is to highlight the traditional actions of herbal plants used by inborn Yanadi community of Seshachalam Biosphere Reserve, Eastern Ghats of Andhra Pradesh, India.

Methods: The ethnobotanical field survey was conducted according to the methods adopted by some authors. In-depth interviews, interactions were conducted with tribal physicians of Yanadi, Nakkala and Irula as well as other tribes practicing and experiencing the use of plant-based medicine. A normal inquiry form was used to gather the appropriate data on herbal plants and their usage of inborn people's lifestyle. Extensive consultations among local people and detailed documentation of the usage of plants were carried out 
in 2014-2017. The aged outmoded opinions and imposts of indigenous people conceded on by word of opening were documented.

Results: A total of 266 medicinally used plant species belonging to 216 genera and 88 families were recognized with help of inborn herbal healers. The study also chronicled the mode of herbal arrangements, mode of the use of herbal plants in various disorders. The study exposed that native people of Seshachalam Biosphere Reserve have good medicinal information and also have preserved plant-based medicinal system of their ascendants used all their diseases. Most of medicinal plants are used in the treatment of indigestion, snake bite and skin diseases. The authors feel that this type of study certainly helps identify ethnic leads for drug development in future.

Conclusions: The ethnobotanical investigation of Seshalam Biosphere area has revealed that the tribes possess good knowledge on plant-based medicine but as they are towards in advanced exposure to transformation, their information on traditional uses of plants is slowly getting eroded. The authors plead for intensive crosscultural studies involving all ethnic tribes in the country for prioritizing or short listing of ethnic leads for various disorders for ultimately developing global level drugs for human welfare and economy development.

Key words: ethnomedicine, Yanadis, Seshachalam Biosphere Reserve, Eastern Ghats, herbal plants, drug development

Słowa kluczowe: medycyna ludowa, Yanadis, Rezerwat Biosfery Seshachalam, Wschodni Ghats, rośliny zielarskie, opracowywanie leków

\section{INTRODUCTION}

Ethnobotany is the study of medicinal plants used by local people, with a particular importance on oldstyle tribal beliefs and information. The World Health Organization (WHO) estimates that unevenly, $80 \%$ of the people from developed and developing countries depend on traditional medicines, especially on plantbased medicine in primary healthcare. The current pharmacopoeia has at least 25\% drugs derived from medicinal herbs and others which are synthetic compounds isolated from herbs. The day to day demand for herbal plants is growing both in developed and developing countries, the recognition of natural harvests is growing, due to the fact that they have small side-effects and are easily accessible at reasonable prices. They are the main source in primary healthcare of poor people. The Indian people have incredible desire for herbal plants and use them in the wide range of applications from cold to mortal diseases.

The recent estimates show that there are about 25000 active plant-based preparations used in traditional medicine and known to remote communities all over from India and the records stated that nearly 10000 medicated formulations are available in the Aboriginal medicinal texts [1].

The indigenous facts concerning herbal plants and their used by traditional healers by way of crude drug development in the present is not only suitable for preservation of cultural diversity and biodiversity for community health care for all the local people.

Nowadays, there is an increasing trend of erosion of traditional knowledge and associated biodiversity due to various reasons, particularly the programs on the upliftment of the Adivasi people throughout the country. A serious lack of protecting the old traditional knowledge as well as the unique flora in the region is observed in this and other study areas of Eastern Ghats. Thus, the present ethnobotanical investigation plays significant role for maintenance and documentation of the traditional knowledge of Yanadi tribe forests of Andhra Pradesh and also advocates the sustainable utilization of these herbal plants.

The anthropology of the region is also interesting. The major ethnic communities of the Seshachalam Biosphere reserve and surrounding areas are Yanadi, Nakkala and Irula and folklore of some village communities. The Yanadis are generally considered into 4 endogamous sub-groups on the basis of livelihoods, i.e. Paki yanadis, Reddi yanadis, Challa yanadis, Adavi yanadis. The yanadis are inland fishermen. They watch and ward the fields of higher caste. Generally, they are settled near canals and tank bunds. The past ethnobotanical works in Seshachalam Biosphere reserve were carried out in $1985-2000$ [2-8] and the present study is one more in this direction. It lists many additional ethnobotanical uses in the region. Also, ethonobotanically 
the surveyed area has not been adequately codified and no comprehensive ethnobotanical account on Yanadi, Nakkala and Irula folklore survey are available on the use of medicinal plants.

The present study was carried out in 2014-2017. The senior author has focused mainly on new applications of traditional medicines and arriving at ethnic leads that may form the foundation for future drug development. Nowadays, there is a need for rapid listing and arranging the ethnic leads for exact ailment by cris-crossing of information through multicultural studies among dissimilar folkloric tribes within a country and then comparing the same with the developed and developing countries in the region for penetrating bio-prospecting and drug development. This calls for collaborative research programs aiming at drug development by all developing countries having rich heritage of ethnic knowledge within the framework of the Rio Convention. The use of a specific species for the same ailment by different unrelated ethnic groups certainly may indicates that the efficacy of probable plants for drug development. Though, scarcity of field taxonomists and ethnobotanists, inadequate financial support for ethnobotanical investigations, lack of co-operation between biotechnologists and ethnobotanists in Bio-prospecting programs on ethnobotanical leads, lack of complete ethnobotanical databases among biodiversity rich countries for proportional ethnobotanical study are shown to be some of the major restrictions in this direction. Therefore, the impending ethnobotanical studies must focus not only on inventories of ethnic knowledge of Adivasi people and development of data bases on ethnic knowledge (for purposes of sharing royalties, if any) but also focus on preservation and regeneration of the traditional beliefs, maintenance of the traditional knowledge against mismanagement.

\section{MATERIALS AND METHODS}

\section{Study area}

Seshachalam Biosphere reserve with $4756 \mathrm{~km}^{2}$ external area is located between $13^{\circ} 30^{\prime}$ and $14^{\circ} 11^{\prime} \mathrm{N}$ and $79^{\circ} 030^{\prime \prime}$ and $79^{\circ} 30^{\prime} \mathrm{E}$ with an altitude reaching from 135 to $1187 \mathrm{~m}$ on southern plateaus of Tellaralla Penta (fig. 1). The variety of wildlife as well as diverse environments and landscapes, the area was selected as Seshachalam Biosphere reserve at $20^{\text {th }}$ Sep, 2011. Seshachalam Biosphere reserve comprises world famous sacred shrines collectively called as Tirumala hills are the abode of Lord Venkateswara or Balaji. These hills are situated at $900 \mathrm{~m}$ above MSL. The sanctum sanctorum is situated on the top of seven adjoining hills, which are believed to be an incarnation of Ananta Sesha, the serpent god. Tirumala hills cover about 365 theerthams consisting of seasonal and perennial waterfalls and springs. Talakona is the highest waterfall (270 ft tall) in the State of Andhra Pradesh.

The topography, deep unreachable valleys, and upright cliffs are among the attractive landscapes and physical formations of Seshachalam Biosphere Reserve. The soils are formed of lateritic, gneissic and quartzite material. Much of the hilly area is composed of granite complex. Usually the entire hill range has a dry climate. The higher elevation of the hill ranges is comparatively cooler than the foot hills. During summer, the climate in places like on the top of the Talakona and Tirumala is cool and pleasant. Hot season is in March and May. The monthly average minimum temperature varies from $18^{\circ} \mathrm{C}$ to $22.67^{\circ} \mathrm{C}$, the lowest in January; maximum $33.36^{\circ} \mathrm{C}$ to $36.21^{\circ} \mathrm{C}$, the highest in May. The Seshachalam Biosphere reserve was experienced with the North-East monsoon (October-December) and Southwest monsoon (June-September). The annual rainfall varies between 569.43 and $1230.81 \mathrm{~mm}$.

The forests of the Seshachalam Biosphere reserve can be broadly categorized into three types: dry deciduous mixed type with patches of moist deciduous forests and scrub type [9]. Dry deciduous forests dominate in the study area. The most dominant species in the Reserve are Anogeissus latifolia, Chloroxylon swietenia, Decaschistia crotonifolia, Pterocarpus santalinus, Phoenix sylvestris, P. loureiroi, Syzygium alternifolium, Terminalia pallida etc. The study area encompasses two protected areas: Sri Venkateswara Wildlife Sanctuary and Sri Venkateswara National Park and large number of sacred groves.

\section{Methodology}

The entire area of Seshachalam Biosphere Reserve is thoroughly studied by repeated surveys in different seasons of the year from 2014 to 2017 covering pre-monsoon, monsoon and post-monsoon seasons. The ethnobotanical field survey was conducted according to the methods adopted by [10-12]. In-depth interviews interactions were conducted with the tribal physicians of Yanadi, Nakkala and Irula and other tribes who practice and experience plant-based medicine in use. A total of 86 informers interviewed were 32 women and 54 men, aged 


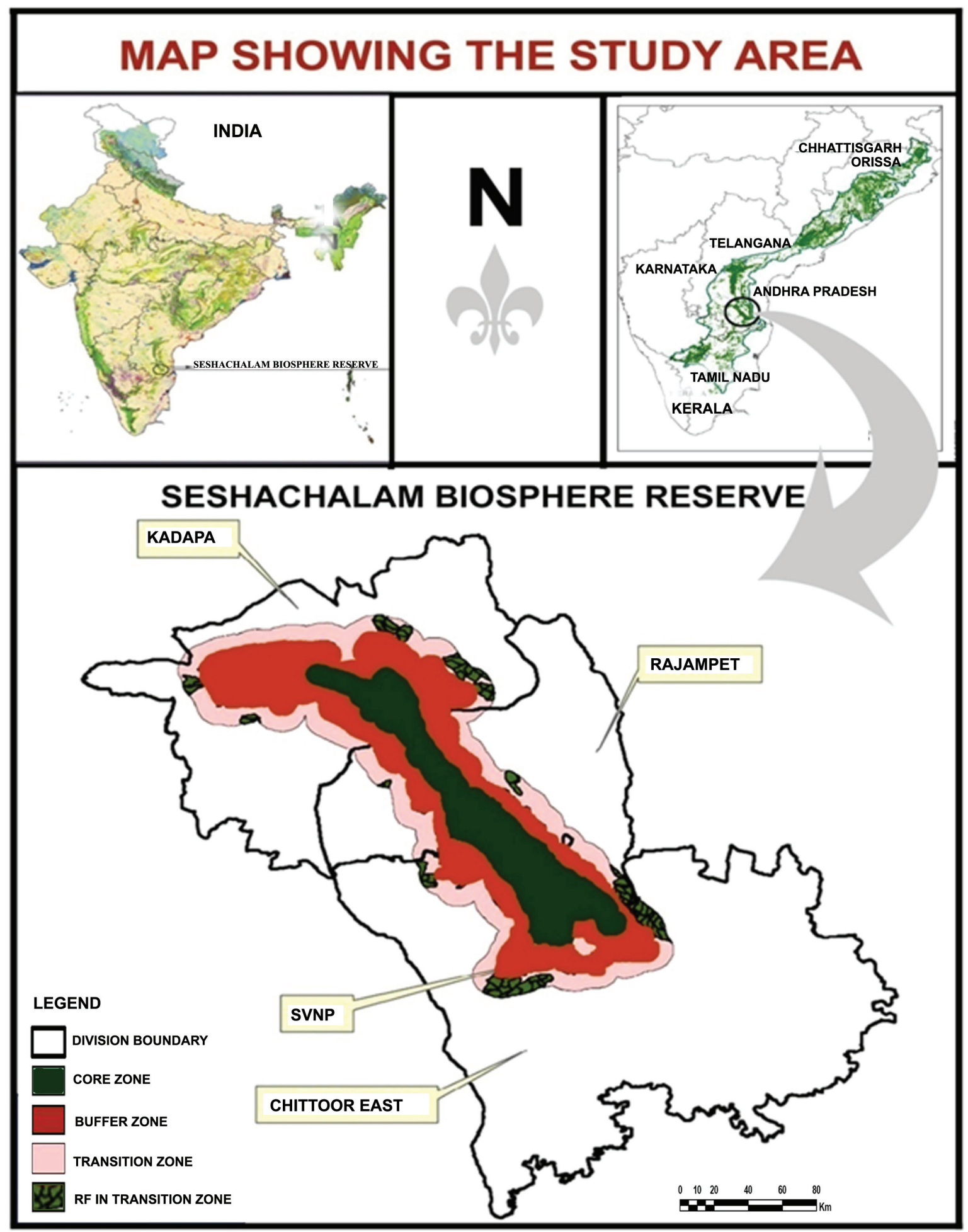

Figure 1

Study area 
50 to 75 in their villages. The information recorded was about the use of medicinal plants, preparation and mode of administration in and around of Seshachalam Biosphere Reserve. The information on these plant species were gathered with reference to the local names. Plant specimens were collected processed for herbarium and identified in accordance with the methodology provided by [13]. The collected specimens were identified with the help of the regional and local floras $[14,15]$. The voucher specimens were deposited in the Herbarium, Department of Botany, Yogi Vemana University, YSR Kadapa (Dist.), Andhra Pradesh, India for future reference.

\section{Enumeration}

In the enumeration of the ethnobotanically important medicinal plants, the species names are alphabetically arranged. This is followed by family name (according to APG classification) in parenthesis, habit, common name, detailed data on traditional uses as provided in tablel.

Ethical approval: The conducted research is not related to either human or animal use.

Table 1

Significant medicinal plants of Seshachalam Biosphere Reserve in Eastern Ghats

\begin{tabular}{|c|c|c|c|c|c|}
\hline No & Botanical name & Family & Habit & Vernacular name & Medicinal uses \\
\hline 1 & Abrus precatorius L. & Fabaceae-F & climber & Gurivinda & anti-inflamatory \\
\hline 2 & Abutilon hirtum (Lam.) Sweet & Malvaceae & shrub & Nela benda & nervous debility, aphrodisiac \\
\hline 3 & Abutilon indicum (L.) Sweet & Malvaceae & shrub & $\begin{array}{l}\text { Duvvenakaya/ } \\
\text { Tutturubenda }\end{array}$ & haematuria \\
\hline 4 & Acacia catechu (L.f.) Willd. & Fabaceae-M & tree & Chandra, Sandra & diabetes, cough, cold, leucoderma \\
\hline 5 & Acacia leucophloea (Roxb.) Willd. & Fabaceae-M & tree & Tella tumma & diuretic \\
\hline 6 & Acalypha indica $\mathrm{L}$. & Euphorbiaceae & herb & Muripinda & $\begin{array}{l}\text { intestinal worms, stomach aches, } \\
\text { rheumatism, bronchitis, asthma, scabies } \\
\text { and skin diseases }\end{array}$ \\
\hline 7 & Achyranthes aspera L. & Amaranthaceae & herb & Uttareni & diuretic, piles \\
\hline 8 & Actinopteris radiata (Sw.) Link. & Actinopteridaceae & herb & Mayuri shika & skin diseases \\
\hline 9 & Adiantum caudatum L. & Adiantaceae & herb & Raja hamsa & diabetes \\
\hline 10 & Aegle marmelos (L.) Corrêa & Rutaceae & shrub & Maredu/Bilva & dysentery \\
\hline 11 & Aerva lanata (L.) Juss. & Amaranthaceae & herb & Pindikura & urinary diseases \\
\hline 12 & Ageratum conyzoides (L.) L. & Asteraceae & herb & Goat weed & nervine tonic \\
\hline 13 & Alangium salviifolium (L. f.) Wangerin & Cornaceae & tree & Udaga / Ankolamu & dog bite \\
\hline 14 & Albizia odoratissima (L.f.) Benth & Fabaceae-M & tree & Chinduga & leprosy \\
\hline 15 & Albizia thompsonii Brandis & Fabaceae-M & tree & Velugu Chinta. & skin diseases, ulcers \\
\hline 16 & Albizia amara (Roxb.) B.Boivin & Fabaceae-M & tree & Cheekireni & inflammation \\
\hline 17 & Albizia lebbeck (L.) Benth. & Fabaceae-M & tree & $\begin{array}{l}\text { Dirsenmu, } \\
\text { Dirasana }\end{array}$ & $\begin{array}{l}\text { bites and stings, blood purification, skin } \\
\text { problems }\end{array}$ \\
\hline 18 & Alstonia scholaris (L.) R. Br. & Apocynaceae & climber & Edakulapala & galactagogue, asthma \\
\hline 19 & $\begin{array}{l}\text { Andrographis paniculata (Burm. f.) } \\
\text { Nees }\end{array}$ & Acanthaceae & herb & Nelavemu & fever \\
\hline 20 & Anisomelea malabarica (L.) & Lamiaceae & shrub & Moga-Bira & eczema, diarrhea \\
\hline 21 & Annona squamosa L. & Annonaceae & tree & Sitapalem & abortifacient \\
\hline 22 & $\begin{array}{l}\text { Anogeissus latifolia (Roxb. ex Dc.) Wall. } \\
\text { ex Guillem. \& Perr. }\end{array}$ & Combretaceae & tree & Chirimanu/Elama & piles, snake bite \\
\hline 23 & Argemone mexicana L. & Papaveraceae & herb & $\begin{array}{l}\text { Kusuma / } \\
\text { Brahmadandi }\end{array}$ & syphilis \\
\hline
\end{tabular}




\begin{tabular}{|c|c|c|c|c|c|}
\hline 24 & Aristolochia bracteolata Lam. & Aristolochiaceae & herb & Gadidagadapa & eczema, leprosy \\
\hline 25 & Aristolochia indica $\mathrm{L}$. & Aristolochiaceae & herb & Easwari & snake bite \\
\hline 26 & Asparagus racemosus Willd. & Asparagaceae & herb & Sathavari & diabetes, leucorrhoea \\
\hline 27 & Atalantia monophylla DC. & Rutaceae & shrub & Munukudu & antiseptic, fever \\
\hline 28 & Azadirachta indica A. Juss. & Meliaceae & tree & Vepa & skin diseases \\
\hline 29 & Azima tetracantha Lam. & Salvadoraceae & shrub & Tella uppili & leprosy, eczema \\
\hline 30 & $\begin{array}{l}\text { Baccharoides anthelmintica (L.) } \\
\text { Moench (=Vernonia anthelmintica L.) }\end{array}$ & Asteraceae & herb & Adavi jeelakarra & digestion \\
\hline 31 & Bacopa monnieri (L.) Wettst. & Plantaginaceae & herb & Brahmi & memory booster \\
\hline 32 & Balanites aegyptiaca (L.) Delile & Zygophyllaceae & tree & Gara & anthelmintic, asthma, dysentery, epilepsy \\
\hline 33 & Basella alba $\mathrm{L}$. & Basellaceae & climber & Bachali & constipation \\
\hline 34 & Bauhinia racemosa Lam. & Fabaceae- $C$ & tree & Are fibres & malaria, fever \\
\hline 35 & Bauhinia purpurea L. & Fabaceae-C & tree & Deva Kanchana & asthma, cold, cough, rheumatic pain \\
\hline 36 & Benincasa hispida (Thunb) Cogn. & Cucurbitaceae & tree & Gummadi & urinary dysfunction, summer fevers \\
\hline 37 & Biophytum sensitivum (L.) DC. & Oxilidaceae & herb & Puli chinta & $\begin{array}{l}\text { antidote, snake bite, antidiabetic, } \\
\text { antilithic }\end{array}$ \\
\hline 38 & Bixa orellana $\mathrm{L}$. & Bixaceae & tree & Jabaru kaya & gonorrhoea, fever, dysentery jaundice \\
\hline 39 & Blumea axillaris (Lam.) DC. & Asteraceae & herb & Kukkapogaku & dropsy, throat infection \\
\hline 40 & Boerhavia diffusa $\mathrm{L}$. & Nyctaginaceae & herb & Attamamidi & urinary disorders \\
\hline 41 & Bombax ceiba L. & Malvaceae & tree & Adavi Buruga & diabetes, diuretic \\
\hline 42 & Borassus flabellifer $\mathrm{L}$. & Arecaceae & tree & Tati & oedema, constipation \\
\hline 43 & $\begin{array}{l}\text { Boswellia ovalifoliolata N. P. Balakr. \& } \\
\text { A. N. Henry }\end{array}$ & Burseraceae & tree & Sambrrani & stomach ulcers, dysentery \\
\hline 44 & Boswellia serrata Roxb. ex Colebr. & Burseraceae & tree & Sambrani & arthritis \\
\hline 45 & $\begin{array}{l}\text { Buchnania axilaris (Desr.) } \\
\text { Ramamoorthy }\end{array}$ & Anacardiaceae & tree & Sara & boils, cardio tonic, wounds \\
\hline 46 & Butea monosperma (Lam) Taub. & Fabaceae-F & tree & Moduga & jaundice, astringent \\
\hline 47 & Byttneria herbacea Roxb. & Malvaceae & herb & Magasirigadda & swelling of legs \\
\hline 48 & Cadaba fruticosa (L.) Druce & Capparaceae & shrub & Adamorinika & indigestion, fever \\
\hline 49 & Caesalpinia bonduc (L.) Roxb. & Fabaceae-F & shrub & Gacha & leucorrhoea, hydrocele \\
\hline 50 & Calophyllum inophyllum $\mathrm{L}$. & Clusiaceae & tree & Ponna & rheumatism, astringent \\
\hline 51 & Capparis divaricata Lam. & Capparaceae & tree & Guda remidi & $\begin{array}{l}\text { antiseptic, asthma, post deliver } \\
\text { complaints }\end{array}$ \\
\hline 52 & Capparis grandis L. f. & Capparaceae & tree & $\begin{array}{l}\text { Adavi Booda } \\
\text { Remidi }\end{array}$ & aphrodisiac, wounds, ulcer, scorpion sting \\
\hline 53 & Capparis sepiaria $\mathrm{L}$. & Capparaceae & shrub & Nalla uppili & febrifuge \\
\hline 54 & Capparis zeylanica L. & Capparaceae & shrub & Adonda & antihelmenthic, sedative \\
\hline 55 & Caralluma adscendens (Roxb.) R. Br. & Apocynaceae & herb & Kundeti chevi & $\begin{array}{l}\text { ear infections, applied to teeth to treat } \\
\text { caries, bites and stings }\end{array}$ \\
\hline 56 & Cardiospermum halicacabum L. & Sapindaceae & climber & Buddakakara & rheumatism, nervous disorders \\
\hline 57 & Careya arborea Roxb. & Lecythidaceae & tree & Budda darimi & eye diseases, skin sores \\
\hline 58 & Carissa carandas $\mathrm{L}$. & Apocynaceae & shrub & Vaka & diabetes, stomachic \\
\hline 59 & Cassia fistula L. & Fabaceae-C & tree & Rela & diabetes \\
\hline 60 & $\begin{array}{l}\text { Cassia italica (Mill.) Lam. ex F.W. } \\
\text { Andrews }\end{array}$ & Fabaceae-C & herb & Nelatangedu & bone fracture \\
\hline
\end{tabular}




\begin{tabular}{|c|c|c|c|c|c|}
\hline 61 & Cassine glauca (Rottb.) Kuntze & Celastraceae & tree & Nerdhi & snake bite, dysuria \\
\hline 62 & Cassytha filiformis $\mathrm{L}$. & Lauraceae & climber & Sitamma savaralu & dysentery, hair tonic \\
\hline 63 & $\begin{array}{l}\text { Catunaregam spinosa (Thunb.) } \\
\text { Tirveng. }\end{array}$ & Rubiaceae & shrub & Manga & diarrhea, astringent \\
\hline 64 & Centella asiatica (L.) Urban. & Apiaceae & herb & Saraswathi & brain tonic \\
\hline 65 & Ceropegia spiralis Wight & Apocynaceae & herb & Nimmati gadda. & indigestion \\
\hline 66 & $\begin{array}{l}\text { Chamaecrista absus (L.) H. S. Irwin\& } \\
\text { Barneby (= Cassia absus L.) }\end{array}$ & Fabaceae-C & herb & Chanupala vittulu & constipation, cough \\
\hline 67 & $\begin{array}{l}\text { Cheilocostus speciosus (J. Koenig) C. D. } \\
\text { Specht (= Costus speciosus) }\end{array}$ & Costaceae & herb & $\begin{array}{l}\text { Adavi allam/ } \\
\text { Chengalva cost }\end{array}$ & dyspepsia, snake bite \\
\hline 68 & Chloroxylon swietenia DC. & Rutaceae & tree & Billudu. & $\begin{array}{l}\text { epilepsy, scorpion sting, snake bite, } \\
\text { wounds, ulcers, mosquito repellent, } \\
\text { urinary problems, rheumatism }\end{array}$ \\
\hline 69 & Cipadessa baccifera (Roth) Miq. & Meliaceae & shrub & Ranaberi & diabetes, wounds \\
\hline 70 & Cissampelos pareira L. & Menispermaceae & shrub & Visha boddi & dropsy, diabetes \\
\hline 71 & Cissus quadrangularis L. & Vitaceae & herb & Nalleru & leucorrhoea, piles \\
\hline 72 & Cissus vitiginea $\mathrm{L}$. & Vitaceae & climber & Adavi gummidi & wounds \\
\hline 73 & Citrullus colocynthis (L.) Schrad. & Cucurbitaceae & climber & Papara & rheumatism, jaundice \\
\hline 74 & Cleome felina L. f. & Cleomaceae & herb & Erra vominta & vermifuge, epistaxis, astringent \\
\hline 75 & Cleome gynandra L. & Cleomaceae & herb & Vaminta & leaf headache, infant paralysis \\
\hline 76 & Cleome monophylla L. & Cleomaceae & herb & Gaddi vaminta. & $\begin{array}{l}\text { restore consciousness, breast ulcer, } \\
\text { galactogogue }\end{array}$ \\
\hline 77 & Cleome viscosa $\mathrm{L}$. & Cleomaceae & herb & Kukkavaminta & $\begin{array}{l}\text { earache, constipation, fever, diarrhea, } \\
\text { headache }\end{array}$ \\
\hline 78 & Coccinia grandis (L.) Voigt & Cucurbitaceae & climber & Kakidonda & diabetes \\
\hline 79 & Cocculus hirsutus (L.) W. Theob. & Menispermaceae & climber & Dusari Teega & rheumatism \\
\hline 80 & Cochlospermum religiosum (L.) Alston & Cochlospermaceae & tree & Konda gogu & dysentery, gonorrhoea \\
\hline 81 & Coldenia procumbens L. & Boraginaceae & herb & Hamsapadu & rheumatism \\
\hline 82 & $\begin{array}{l}\text { Commiphora caudata (Wight \& Arn.) } \\
\text { Engl. }\end{array}$ & Burseraceae & $\begin{array}{l}\text { small } \\
\text { Tree }\end{array}$ & Kondamamidi & rheumatism \\
\hline 83 & $\begin{array}{l}\text { Convolvulus prostratus Forssk. (= } \\
\text { Convolvulus pluricalis) }\end{array}$ & Convolvulaceae & herb & Shankhpushpi & chronic cough, anxiety \\
\hline 84 & $\begin{array}{l}\text { Corallocarpus epigaeus (Rottler) C. B. } \\
\text { Clarke }\end{array}$ & Cucurbitaceae & climber & Mukkudonda & eczema, dysentery \\
\hline 85 & Cordia dichotoma G. Forst. & Boraginaceae & tree & $\begin{array}{l}\text { Bankamanu/ } \\
\text { Nakkera }\end{array}$ & bronchial disorders, fever \\
\hline 86 & Coriandrum sativum $\mathrm{L}$. & Apiaceae & herb & Dhaniyalu & $\begin{array}{l}\text { gastric complaints, small pox, nausea, } \\
\text { anemia, fevers, cold and hernias }\end{array}$ \\
\hline 87 & $\begin{array}{l}\text { Crateva religiosa } \mathrm{G} \text {. Forst. (= Crataeva } \\
\text { magna) }\end{array}$ & Capparaceae & tree & Velimirichettu & $\begin{array}{l}\text { renel, vesical calculi, bronchitis, gastric } \\
\text { problem, skin diseases, fever }\end{array}$ \\
\hline 88 & Crotalaria retusa L. & Fabaceae-F & herb & Sanapusphi & scabies \\
\hline 89 & Curculigo orchioides Gaertn. & Hypoxidaceae & herb & Nelathati & diarrhoea, potency \\
\hline 90 & Curcuma neilgherrensis Wight & Zingiberaceae & herb & Konda Pasupu & $\begin{array}{l}\text { skin diseases, anti-asthmatic, antitumour, } \\
\text { stomachic }\end{array}$ \\
\hline 91 & Curcuma pseudomontana J. Graham & Zingiberaceae & herb & Adavi Pasupu & swellings due to wounds \\
\hline 92 & Cycas beddomi Dyer. & Cycadaceae & tree & Peritha & aphrodisiac \\
\hline 93 & $\begin{array}{l}\text { Cymbopogon flexuosus (Nees ex Steud.) } \\
\text { W. Watson }\end{array}$ & Poaceae & herb & Nimma gaddi & citral oil \\
\hline 94 & $\begin{array}{l}\text { Dalbergia lanceolaria subsp. paniculata } \\
\text { (Roxb.) Thoth. }\end{array}$ & Fabaceae-F & tree & Pacchari & filarial swelling \\
\hline
\end{tabular}




\begin{tabular}{|c|c|c|c|c|c|}
\hline 95 & Dalbergia latifolia Roxb. & Fabaceae-F & tree & Jittagi / Iridi & ulcers, leprosy \\
\hline 96 & Datura metal L. & Solanaceae & herb & Nalla ummetta & epilepsy \\
\hline 97 & Datura stramonium L. & Solanaceae & shrub & Ummetta & asthma, narcotic \\
\hline 98 & Decalepis hamiltonii Wight \& Arn. & Apocynaceae & shrub & Maredu kommulu & haemorrhagia, appetizer \\
\hline 99 & Decaschistia crotonifolia Wight \& Arn. & Malvaceae & shrub & Adavigogu & hydrocele \\
\hline 100 & $\begin{array}{l}\text { Decaschistia cuddapahensis T. K. Paul\& } \\
\text { M. P. Nayar }\end{array}$ & Malvaceae & shrub & Magasiri & aphrodisiac \\
\hline 101 & Deccania pubescens (Roth) Tirveng. & Rubiaceae & tree & Konda manga & sores \\
\hline 102 & Derris scandens (Roxb.) Benth. & Fabaceae-F & climber & Nalla teega & snake bite \\
\hline 103 & Desmodium triflorum (L.) DC. & Fabaceae-F & herb & Munta mandu & galactagogue, diarrhea \\
\hline 104 & Dillenia pentagyna Roxb. & Dilleniaceae & tree & Chinna Kalinga. & hair wash \\
\hline 105 & Dioscorea bulbifera L. & Dioscoreaceae & climber & $\begin{array}{l}\text { Nookala gadda, } \\
\text { Aadasancha }\end{array}$ & increases sexual vigour \\
\hline 106 & Dioscorea pentaphylla L. & Dioscoreaceae & climber & Injedigadda & dysentery, leprosy \\
\hline 107 & Dioscorea pentaphylla L. & Dioscoreaceae & climber & Yeleru tiga & indigestion \\
\hline 108 & Diospyros melanoxylon Roxb. & Ebenaceae & tree & Tumki & dyspepsia, diuretic \\
\hline 109 & Dodonaea viscosa Jacq. & Sapindaceae & shrub & Bandaru & antipyretic, bone fractures \\
\hline 110 & $\begin{array}{l}\text { Dregea volubilis (L. f.) Benth. ex Hook. } \\
\text { f. (=Wattakaka volubilis (L. f.) Stapf) }\end{array}$ & Apocynaceae & climber & Kallisi & snake bite, body pains \\
\hline 111 & Echinops echinatus Roxb. & Asteraceae & herb & MullaBanthi & diuretic, lice eradication \\
\hline 112 & Eclipta prostrata (L.) L. & Asteraceae & herb & Gunta galijeru & asthma, jaundice \\
\hline 113 & $\begin{array}{l}\text { Enicostema axillare (Poir. ex Lam.) A. } \\
\text { Raynal }\end{array}$ & Gentianaceae & herb & Gulividi & scabies, gout, scorpion sting \\
\hline 114 & Eriolaena hookeriana Wight \& Arn. & Malvaceae & tree & $\begin{array}{l}\text { Nara botku, } \\
\text { Gurraputhada }\end{array}$ & wounds, cuts, pains of eyeballs \\
\hline 115 & Erythroxylum monogynum Roxb. & Erythroxylaceae & shrub & Devadari & stomachic, diuretic \\
\hline 116 & Euphorbia hirta L. & Euphorbiaceae & herb & Nanabala & cough, dysentery \\
\hline 117 & Evolvulus alsinoides (L.) L. & Convolvulaceae & herb & Vistikantha & fever \\
\hline 118 & Ficus benghalensis L. & Moraceae & tree & Marri & rheumatism \\
\hline 119 & Ficus microcarpa L.f. & Moraceae & tree & Kondajuvvi & wounds, diabetes \\
\hline 120 & $\begin{array}{l}\text { Firmiana simplex (L.) W. Wight } \\
\text { (=Sterculia urens } \text { Roxb.) }\end{array}$ & Malvaceae & tree & $\begin{array}{l}\text { Tabasi, Thella } \\
\text { poliki }\end{array}$ & $\begin{array}{l}\text { rheumatic pains, ulcers, cooling, } \\
\text { dysentery, anti-diabetic, throat infection, } \\
\text { tooth disorders }\end{array}$ \\
\hline 121 & Flacourtia indica (Burm. f.) Merr. & Salicaceae & tree & Mulielka, Kanregu & cholera \\
\hline 122 & Gardenia gummifera L. f. & Rubiaceae & tree & Bikki & ulcer, constipation \\
\hline 123 & Gardenia resinifera Roth & Rubiaceae & tree & Erribikki & constipation, bronchites \\
\hline 124 & Givotia moluccana (L.) Sreem & Euphorbiaceae & tree & Tella poliki & dandruff, psoriasis, rheumatism \\
\hline 125 & Gloriosa superba L. Sp. Pi. & Colchicaceae & climber & $\begin{array}{l}\text { Nabhi/ } \\
\text { Nagetigadda }\end{array}$ & leprosy; abortifacient \\
\hline 126 & Glycosmis pentaphylla (Retz.) DC. & Rutaceae & shrub & Gonji & diabetes; eczema \\
\hline 127 & Gmelina asiatica L. & Lamiaceae & shrub & Adavi Gummadi & dental problems \\
\hline 128 & Grewia damine Gaertn. & Malvaceae & tree & Jana & stomachache for children \\
\hline 129 & Grewia flavescens Juss. & Malvaceae & tree & Banka jana & wounds, boils, dysentery \\
\hline 130 & Grewia hirsuta Vahl. & Malvaceae & shrub & Adavichamanthi & dysentery, antidote to opium poison \\
\hline
\end{tabular}




\begin{tabular}{|c|c|c|c|c|c|}
\hline 131 & Grewia tiliaefolia Vahl. & Malvaceae & tree & $\begin{array}{l}\text { Tella jana, } \\
\text { Charachi, Tada }\end{array}$ & $\begin{array}{l}\text { aphrodisiac, cough, diarrhoea, } \\
\text { spermatorrhoea, antidandruff, skin } \\
\text { diseases }\end{array}$ \\
\hline 132 & Gymnema sylvestre (Retz.) R. Br. ex Sm. & Apocynaceae & $\begin{array}{l}\text { woody } \\
\text { vine }\end{array}$ & Podapatri & diabetes \\
\hline 133 & Gyrocarpus americanus Jacq. & Hernandiaceae & tree & Taniki/Nalla poliki & cancer \\
\hline 134 & Habenaria roxburghii Nicolson & Orchidaceae & herb & Leena Gadda & wounds \\
\hline 135 & Haldina cordifolia (Roxb.) Ridsdale & Rubiaceae & tree & Rudra ganapa & stomachic \\
\hline 136 & Hardwickia binata Roxb. & Fabaceae-F & tree & Yepi & rheumatism \\
\hline 137 & Helicteres isora $\mathrm{L}$. & Malvaceae & shrub & Gooba thada & diabetes, dysentery \\
\hline 138 & Heliotropium indicum L. & Boraginaceae & herb & Nagadanthi & ulcers, eczema \\
\hline 139 & $\begin{array}{l}\text { Hemidesmus indicus (L.) R. Br. ex } \\
\text { Schult. }\end{array}$ & Apocynaceae & herb & Sugandhapala & cooling beverage, cardio tonic \\
\hline 140 & Hemionitis arifolia (Burm. f.) Moore & Hemionitidaceae & herb & Rama bhanam & antidiabetic \\
\hline 141 & Hibiscus platanifolius (Willd.) Sweet & Malvaceae & tree & Kondagogu & diabetes, rheumatism \\
\hline 142 & $\begin{array}{l}\text { Hibiscus micranthus L. f. (=H. } \\
\text { ovalifolius (Forsk.) Vahl. }\end{array}$ & Malvaceae & herb & $\begin{array}{l}\text { Nithyamalli, } \\
\text { Geesu. }\end{array}$ & gonorrhoea, leucorrhoea \\
\hline 143 & Hiptage benghalensis (L.) Kurz & Malphigiaceae & climber & Madhavi tega & diarrhea, dysentery \\
\hline 144 & $\begin{array}{l}\text { Holarrhena pubescens Wall. ex G. Don } \\
(=\text { H. antidysenterica })\end{array}$ & Apocynaceae & tree & $\begin{array}{l}\text { Kola musthi/pala/ } \\
\text { kodisapala }\end{array}$ & diarrohoea, antidysentric \\
\hline 145 & Holostemma ada-kodien Schult. & Apocynaceae & climber & $\begin{array}{l}\text { Tella jilledu/ } \\
\text { Peyyibaddu }\end{array}$ & gonorrhea, diabetes \\
\hline 146 & Hugonia mystax L. & Linaceae & shrub & Kakibeera & antihelmenthic \\
\hline 147 & Hybanthus enneaspermus (L.) F. Muell. & Violaceae & herb & Ratna purusha & leucorrhoea, diabetes \\
\hline 148 & Ichnocarpus frutescens (L.) W. T. Aiton & Apocynaceae & climber & Palateega & blood purifier \\
\hline 149 & Impatiens balsamina $\mathrm{L}$. & Balsaminaceae & herb & $\begin{array}{l}\text { Chilakamukku } \\
\text { puvvu, Kasi } \\
\text { tumma }\end{array}$ & $\begin{array}{l}\text { cooling, diuretic, improve blood } \\
\text { circulation, intestinal neuralgia }\end{array}$ \\
\hline 150 & Ixora pavetta Andr. & Rubiaceae & tree & Korivi/Papidi & dysentery, urinary disorders \\
\hline 151 & Jasminum auriculatum Vahl. & Oleaceae & climber & Adavimalli & dropsy \\
\hline 152 & Justicia adhatoda L. & Acanthaceae & shrub & Addasaram & diabetes, fever \\
\hline 153 & Kydia calycina Roxb. & Malvaceae & tree & Bottuka & skin diseases and ulcers, wounds, cough \\
\hline 154 & Lannea coromandelica (Houtt.) Merr. & Anacardiaceae & tree & Gumphena & ulcers, dental diseases \\
\hline 155 & Lantana camara L. & Verbenaceae & shrub & Phallikampa & tooth ache, wounds \\
\hline 156 & Lawsonia inermis $\mathrm{L}$. & Lythraceae & tree & Gorintaku & diarrhea, diabetes \\
\hline 157 & $\begin{array}{l}\text { Ledebouria revoluta (L. f.) Jessop (= } \\
\text { Scilla hyacinthina) }\end{array}$ & Liliaceae & herb & Nakkeragadda & leprosy, diuretic \\
\hline 158 & Leonotis nepetifolia (L.) R. Br. & Lamiaceae & herb & Ranabheri & rheumatism \\
\hline 159 & Lepidagathis cristata Willd. & Acanthaceae & herb & $\begin{array}{l}\text { Nakka pidi, } \\
\text { Lankapindi }\end{array}$ & burns, wounds \\
\hline 160 & Lepisanthes tetraphylla Radlk. & Sapindaceae & tree & Sali kunkudu & skin diseases \\
\hline 161 & $\begin{array}{l}\text { Leptadenia reticulata (Retz.) Wight \& } \\
\text { Arn. }\end{array}$ & Apocynaceae & climber & Mukkupalateega & abortifacient \\
\hline 162 & Limnophila indica (L.) Druce & Plantaginaceae & herb & Sambrani & antiseptic, dysentery \\
\hline 163 & Limonia acidissima Groff & Rutaceae & tree & Velaga & stomachic, astringent \\
\hline 164 & $\begin{array}{l}\text { Madhuca longifolia (J. König ex L.) J. } \\
\text { F. Macbr. }\end{array}$ & Sapotaceae & tree & Ippa & cough, skin diseases \\
\hline
\end{tabular}




\begin{tabular}{|c|c|c|c|c|c|}
\hline 165 & Maerua oblongifolia (Forssk.) A. Rich. & Capparaceae & tree & Magasirigadda & aphrodisiac \\
\hline 166 & Mallotus philippensis (Lam.) Müll.Arg. & Euphorbiaceae & tree & Sinduri & syphilis, gonorrhoea \\
\hline 167 & Manilkara hexandra (Roxb.) Dubard & Sapotaceae & tree & Pala & headache \\
\hline 168 & $\begin{array}{l}\text { Maytenus emarginata (Ruiz \& Pav.) } \\
\text { Loes. }\end{array}$ & Celastraceae & shrub & Danthi & mouth ulcers \\
\hline 169 & Melochia corchorifolia L. & Malvaceae & herb & Siltantakoora & snake bite \\
\hline 170 & Mentha piperita L. & Lamiaceae & herb & $\begin{array}{l}\text { Including in the } \\
\text { text }\end{array}$ & including in the text \\
\hline 171 & Mimosa pudica L. & Fabaceae-M & herb & Aathipathi & constipation, leprosy \\
\hline 172 & Mimusops elengi L. & Sapotaceae & tree & Pogada & diarrhea \\
\hline 173 & Morinda pubescens Sm. & Rubiaceae & tree & $\begin{array}{l}\text { Mulugu chettu, } \\
\text { Togaru chettu }\end{array}$ & rheumatic diseases \\
\hline 174 & Moringa oleifera Lam. & Moringaceae & tree & Munaga & $\begin{array}{l}\text { diarrhea and gastric ulcers, bronchitis, } \\
\text { fevers, ear and eye infections }\end{array}$ \\
\hline 175 & Mucuna pruriens (L.) DC. & Fabaceae-F & climber & Pativratha & tumors on body \\
\hline 176 & Murraya paniculata (L.) Jack & Rutaceae & tree & Naramusti & snake bite, dropsy \\
\hline 177 & Naravelia zeylanica (L.) DC. & Ranunculaceae & climber & Pulla bachala & $\begin{array}{l}\text { headache, leprosy, rheumatic, skin } \\
\text { diseases, toothache }\end{array}$ \\
\hline 178 & Nelumbo nucifera Gaertn. & Nelumbonaceae & herb & Tamara & $\begin{array}{l}\text { diarrhea, cardiac debility, leprosy, } \\
\text { aphrodisiac, leucoderme }\end{array}$ \\
\hline 179 & Nymphaea nouchali Burm. f. & Nymphaeaceae & herb & Kaluva & $\begin{array}{l}\text { diarrhea, dysentery, cardio tonic, } \\
\text { aphrodisiac }\end{array}$ \\
\hline 180 & Ochna obtusata DC. & Ochnaceae & shrub & Errijambi & constipation; asthma \\
\hline 181 & Ocimum tenuiflorum L.(=O. sanctum L.) & Lamiaceae & herb & Nalla tulasi & Ringworm, stimulant, stomachic \\
\hline 182 & Olax scandens Roxb. & Olacaceae & climber & Mekabanda & anaemia; fevers \\
\hline 183 & Oldenlandia corymbosa $\mathrm{L}$. & Rubiaceae & herb & Vermela - vemu & diarrhoea; stomache \\
\hline 184 & Oxalis corniculata $\mathrm{L}$. & Oxilidaceae & herb & Pulichinta & anaemia, cancer, warts \\
\hline 185 & Pavonia zeylanica Cav. & Malvaceae & herb & $\begin{array}{l}\text { Adavi puttudu/ } \\
\text { Chiru benda }\end{array}$ & blood motions \\
\hline 186 & Pavonia odorata Willd. & Malvaceae & herb & Gugu chettu & nervous debility \\
\hline 187 & Pentanema indicum (L.) Ling & Asteraceae & herb & Aggikoora chettu & insect sting \\
\hline 188 & Pentatropis capensis (L. f.) Bullock & Apocynaceae & climber & Yedupullateega & refrigerant \\
\hline 189 & Pergularia daemia (Forssk.) Chiov. & Apocynaceae & climber & Dushtapaku & jaundice, asthma \\
\hline 190 & Phoenix loureiroi Kunth & Arecaceae & tree & Eetha & dysentery, ulcers \\
\hline 191 & $\begin{array}{l}\text { Phyllanthus amarus Schumach. \& } \\
\text { Thonn. }\end{array}$ & Phyllanthaceae & herb & Nelausiri & galactogogue, jaundice \\
\hline 192 & Phyllanthus emblica L. & Phyllanthaceae & tree & Nelli/Usiri & febrifuge, astringent \\
\hline 193 & Phyllanthus indofischeri Bennet & Phyllanthaceae & shrub & Konda Usirika & dyspepsia, jaundice \\
\hline 194 & Phyllanthus urinaria L. & Phyllanthaceae & herb & Uchi usiri & Jaundice, anaemia \\
\hline 195 & Physalis angulata L. & Solanaceae & herb & Budda kodisha. & wounds \\
\hline 196 & $\begin{array}{l}\text { Pimpinella tirupatiensis N. P. Balakr. \& } \\
\text { Subram }\end{array}$ & Apiaceae & herb & Konda Kottimeera & scorpion sting \\
\hline 197 & Pithecellobium dulce (Roxb.) Benth. & Fabaceae- $M$ & tree & Simachintha & leprosy, diabetes \\
\hline 198 & Plumbago zeylanica L. & Plumbaginaceae & herb & Tella chitramulam & scabies, ulcers \\
\hline 199 & $\begin{array}{l}\text { Polyalthia cerasoides (Roxb.) Benth. \& } \\
\text { Hook. f. ex Bedd. }\end{array}$ & Annonaceae & tree & & aphrodisiac \\
\hline
\end{tabular}




\begin{tabular}{|c|c|c|c|c|c|}
\hline 200 & Polycarpaea corymbosa (L.) Lam. & Caryophyllaceae & herb & $\begin{array}{l}\text { Rajuma, } \\
\text { Bonnasara }\end{array}$ & jaundice, kidney diseases \\
\hline 201 & Polygala elongata Klein ex Willd. & Polygalaceae & herb & Nelajenumu & bronchitis, constipation \\
\hline 202 & Pongamia pinnata (L.) Pierre & Fabaceae-F & tree & Kanuga & diabetes, eczema \\
\hline 203 & Portulaca oleracea L. & Portulacaceae & herb & Payalaku. & $\begin{array}{l}\text { gastric trouble, tumors, kidney diseases, } \\
\text { jaundice, diabetes, cephalalgia, toothache, } \\
\text { otalgia }\end{array}$ \\
\hline 204 & Premna tomentosa Willd. & Lamiaceae & tree & Narava/ Namari & dropsy, jaundice \\
\hline 205 & Pterocarpus santalinus L. f. & Fabaceae-F & tree & Rakta chandanam & diabetes, astringent \\
\hline 206 & $\begin{array}{l}\text { Pterospermum xylocarpum (Gaertn.) } \\
\text { Oken }\end{array}$ & Malvaceae & tree & $\begin{array}{l}\text { Lolagu, Chinna } \\
\text { tada }\end{array}$ & leucorrhoea, stomachache \\
\hline 207 & Pueraria tuberosa (Willd.) DC. & Fabaceae-F & climber & Bhoochakra & asthma, rejuvinator \\
\hline 208 & Pupalia lappacea (L.) Juss. & Amaranthaceae & herb & Antudu chettu & toothache \\
\hline 209 & $\begin{array}{l}\text { Rauvolfia serpentina (L.) Benth. ex } \\
\text { Kurz }\end{array}$ & Apocynaceae & herb & Sarpagandha & $\begin{array}{l}\text { Hypertension, psychosis, diabetes, pains, } \\
\text { nervous disorders }\end{array}$ \\
\hline 210 & Rhynchosia beddomei Baker & Fabaceae-F & shrub & Adavi Kandi. & abortifacient \\
\hline 211 & Rhynchosia minima (L.) DC. & Fabaceae-F & tree & Adavichikkudu & abortifacient \\
\hline 212 & Rhynchosia suaveolens (L. f.) DC. & Fabaceae-F & shrub & Karu Kandi & general weakness \\
\hline 213 & Rivea hypocrateriformis Choisy & Convolvulaceae & shrub & Boddi teega & parturition \\
\hline 214 & Salvadora persica L. & Salvadoraceae & tree & $\begin{array}{l}\text { Nalla uppili/ } \\
\text { Varagogu }\end{array}$ & asthma, cough \\
\hline 215 & Santalum album $\mathrm{L}$. & Santalaceae & tree & $\begin{array}{l}\text { Chandanam, } \\
\text { Srigandham }\end{array}$ & diuretic, skin eruptions \\
\hline 216 & Sapindus trifoliatus L. & Sapindaceae & tree & Kunkuduchetta & soap nuts \\
\hline 217 & Sarcostemma acidum (Roxb.) Voigt & Apocynaceae & climber & Pullangi tiga & burning micturition \\
\hline 218 & $\begin{array}{l}\text { Senna alexandrina Mill. (= Cassia } \\
\text { augustifolia) }\end{array}$ & Fabaceae-C & herb & & $\begin{array}{l}\text { piles, intestinal worms, indigestion, } \\
\text { bronchitis }\end{array}$ \\
\hline 219 & Senna montana (Roth) V. Singh & Fabaceae-C & shrub & Pyditangedu & body pains \\
\hline 220 & $\begin{array}{l}\text { Senna obtusifolia (L.) H. S. Irwin\& } \\
\text { Barneby }\end{array}$ & Fabaceae-C & herb & & against vomiting and stomachache \\
\hline 221 & Senna occidentalis (L.) Link & Fabaceae-C & shrub & Kasintha & laxative \\
\hline 222 & Sesbania grandiflora (L.) Pers. & Fabaceae-F & herb & Avisachetta & boils, corysa \\
\hline 223 & Shorea roxburghii G. Don & Dipterocarpaceae & tree & Jalari & astringent, rheumatism \\
\hline 224 & Shorea tumbuggaia Roxb. & Dipterocarpaceae & tree & Tamba/Guggilam & ulcers \\
\hline 225 & Sida acuta Burm. f. & Malvaceae & herb & $\begin{array}{l}\text { Parasukampa, } \\
\text { Polikatta }\end{array}$ & $\begin{array}{l}\text { general debility, nervous debility, } \\
\text { rheumatism }\end{array}$ \\
\hline 226 & Sida cordifolia $\mathrm{L}$. & Malvaceae & herb & Suvana mokka & diabetes, urinary troubles \\
\hline 227 & $\begin{array}{l}\text { Solanum americanum Mill.(= S. nigram } \\
\text { L.) }\end{array}$ & Solanaceae & herb & Kosipalla chetto & vomiting, cough, jaundice \\
\hline 228 & Solanum melongena $\mathrm{L}$. & Solanaceae & shrub & Chiruvanga & hypertension, diabetes \\
\hline 229 & $\begin{array}{l}\text { Solanum virginianum L. }(=S . \\
\text { surrattense Burm. f.) }\end{array}$ & Solanaceae & herb & Errivanga & helminthiasis, tooth problems \\
\hline 230 & Soymida febrifuga (Roxb.) A.Juss. & Meliaceae & tree & Somi & diarrhea, dysentery \\
\hline 231 & Sphaeranthus indicus $\mathrm{L}$. & Asteraceae & herb & Bodasaram & aphrodisiac, anthehelmenthic \\
\hline 232 & Stemona tuberosa Lour. & Stemonaceae & climber & Kanapatige & $\begin{array}{l}\text { night blindness, aphrodisiac, headache, } \\
\text { cough, bodyache }\end{array}$ \\
\hline 233 & Sterculia foetida L. & Malvaceae & tree & Thapasi & diabetes \\
\hline
\end{tabular}




\begin{tabular}{|c|c|c|c|c|c|}
\hline 234 & Strychnos nux-vomica L. & Loganiaceae & tree & Musthi & kidney stones \\
\hline 235 & Strychnos potatorum L. f. & Loganiaceae & tree & Chilla & stomachache \\
\hline 236 & $\begin{array}{l}\text { Suregada lanceolata (Willd.) Kuntze (= } \\
\text { S. augustifolia Baill.) }\end{array}$ & Euphorbiaceae & shrub & Sapranchi & astringent \\
\hline 237 & Syzygium alternifolium (Wight) Walp. & Myrtaceae & tree & Mogi & joint paints \\
\hline 238 & Syzygium cumini (L.) Skeels & Myrtaceae & tree & Neredu & diarrhoea, cough \\
\hline 239 & Tamarindus indica $\mathrm{L}$. & Fabaceae-C & tree & Chinta & indigestion, fever \\
\hline 240 & $\begin{array}{l}\text { Tarenna asiatica (L.) Kuntze ex K. } \\
\text { Schum. }\end{array}$ & Rubiaceae & shrub & Kommi & indigestion \\
\hline 241 & Tephrosia purpurea (L.) Pers. & Fabaceae-F & herb & Vempali & diabetes, ulcers, fever \\
\hline 242 & $\begin{array}{l}\text { Terminalia arjuna (Roxb. ex DC.) } \\
\text { Wight \& Arn. }\end{array}$ & Combretaceae & tree & $\begin{array}{l}\text { Arjuna/Tella } \\
\text { maddi }\end{array}$ & blood motions \\
\hline 243 & Terminalia bellirica (Gaertn.) Roxb. & Combretaceae & tree & Thandra/Tani & dysentery \\
\hline 244 & Terminalia chebula Retz. & Combretaceae & tree & Karaka & cough, piles \\
\hline 245 & Terminalia pallida Brandis & Combretaceae & tree & Tella karaka & diarrhoea \\
\hline 246 & $\begin{array}{l}\text { Thespesia lampas (Cav.) Dalzell \& A. } \\
\text { Gibson }\end{array}$ & Malvaceae & shrub & $\begin{array}{l}\text { Kondapatti, Adavi } \\
\text { patti }\end{array}$ & gonorrhoea, antidiabetic, stomachache \\
\hline 247 & Thespesia populnea (L.) Sol. ex Corrêa & Malvaceae & tree & Gangaravi & boils, ring worms \\
\hline 248 & Tinospora cordifolia (Willd.) Miers. & Menispermaceae & climber & $\begin{array}{l}\text { Tippa teega,Bael } \\
\text { tiga }\end{array}$ & gonorrhea, skin diseases, fever, wounds \\
\hline 249 & Tribulus terrestris $\mathrm{L}$. & Zygophyllaceae & herb & Palleru & aphrodisiac, leprosy \\
\hline 250 & Trichosanthes cucumerina L. & Cucurbitaceae & climber & AdaviPotla & diabetes \\
\hline 251 & Trichosanthes tricuspidata Lour. & Cucurbitaceae & climber & Papara & sores, headache \\
\hline 252 & Trichuriella monsoniae (L. f.) Bennet & Amaranthaceae & herb & Jerrykau & $\begin{array}{l}\text { scorpion sting, headache, boils, sores } \\
\text { wounds, cooling agent }\end{array}$ \\
\hline 253 & Tridax procumbens (L.) L. & Asteraceae & herb & Gaddi chamanthi & dysentery, wounds \\
\hline 254 & Tylophora indica (Burm. f.) Merr. & Apocynaceae & climber & Kakkupala & asthma, epilepsy \\
\hline 255 & Urena lobata L. & Malvaceae & shrub & $\begin{array}{l}\text { Peddabenda, } \\
\text { Nallabenda }\end{array}$ & piles, aphrodisiac, diabetes \\
\hline 256 & $\begin{array}{l}\text { Vanda tessellata (Roxb.) Hook. ex G. } \\
\text { Don. }\end{array}$ & Orchidaceae & herb & $\begin{array}{l}\text { Veduru } \\
\text { bhadhanika }\end{array}$ & bone fracture \\
\hline 257 & Ventilago denticulata Willd. & Rhamnaceae & climber & Surati/Surudu & sprains, malarial fever \\
\hline 258 & Vernonia cinerea (L.) Less. & Asteraceae & herb & Saha devi & fever \\
\hline 259 & Viscum articulatum Burm. f. & Santalaceae & shrub & Badanika & ulcers, febrifuge \\
\hline 260 & Vitex altissima L. f. & Lamiaceae & tree & Nemaliadugu & leprosy \\
\hline 261 & Walsura trifoliolata (A. Juss.) Harms & Meliaceae & tree & Valudu & emmenagogue \\
\hline 262 & Wrightia tinctoria R. Br. & Apocynaceae & tree & Reppala & cough, aphrodisiac \\
\hline 263 & Ximenia americana $\mathrm{L}$. & Olacaceae & shrub & Nakkera & diarrhea, skin diseases, ulcers \\
\hline 264 & Zingiber officinale Roscoe & Zingiberaceae & herb & Allamu & diabetes, cough, asthma \\
\hline 265 & Ziziphus jujuba Mill. & Rhamnaceae & tree & Regu & scorpion sting, diarrhoea \\
\hline 266 & Ziziphus xylopyrus (Retz.) Willd. & Rhamnaceae & tree & Gotti & asthma, aphrodisiac \\
\hline
\end{tabular}




\section{RESULTS AND DISCUSSION}

The study revealed that medicinal properties of 266 species of 216 genera belonging to 88 families used by Yanadi tribe inhabiting parts of Seshachalam Biosphere Reserve (fig. 2, 3). Malvaceae is the dominant family (26 species), followed by Fabaceae-F (18), Apocynaceae (18), Fabaceae-C (10), Asteraceae and Rubiaceae ( 9 species each), FabaceaeM (8), Capparaceae \& Lamiaceae (7 species each),
Cucurbitaceae, Rutaceae \& Solanaceae (6 species each). The remaining 59 families underwritten are of one or two species each. The dominated species are trees (99) followed by herbs (90) shrubs (43) and climbers (34). Of the 266 species, some species like Albizia thompsoni, Boswelia ovalifoliata, Ceropegia spiralis, Cycas beddomi, Pimpinella tirupatiensis, Pterocarpus santalilnus, Rhynchosia beddomei, Shorea tumbeuggaia, Syzygium alternifolium and Terminalia pallida are endemic and threatened

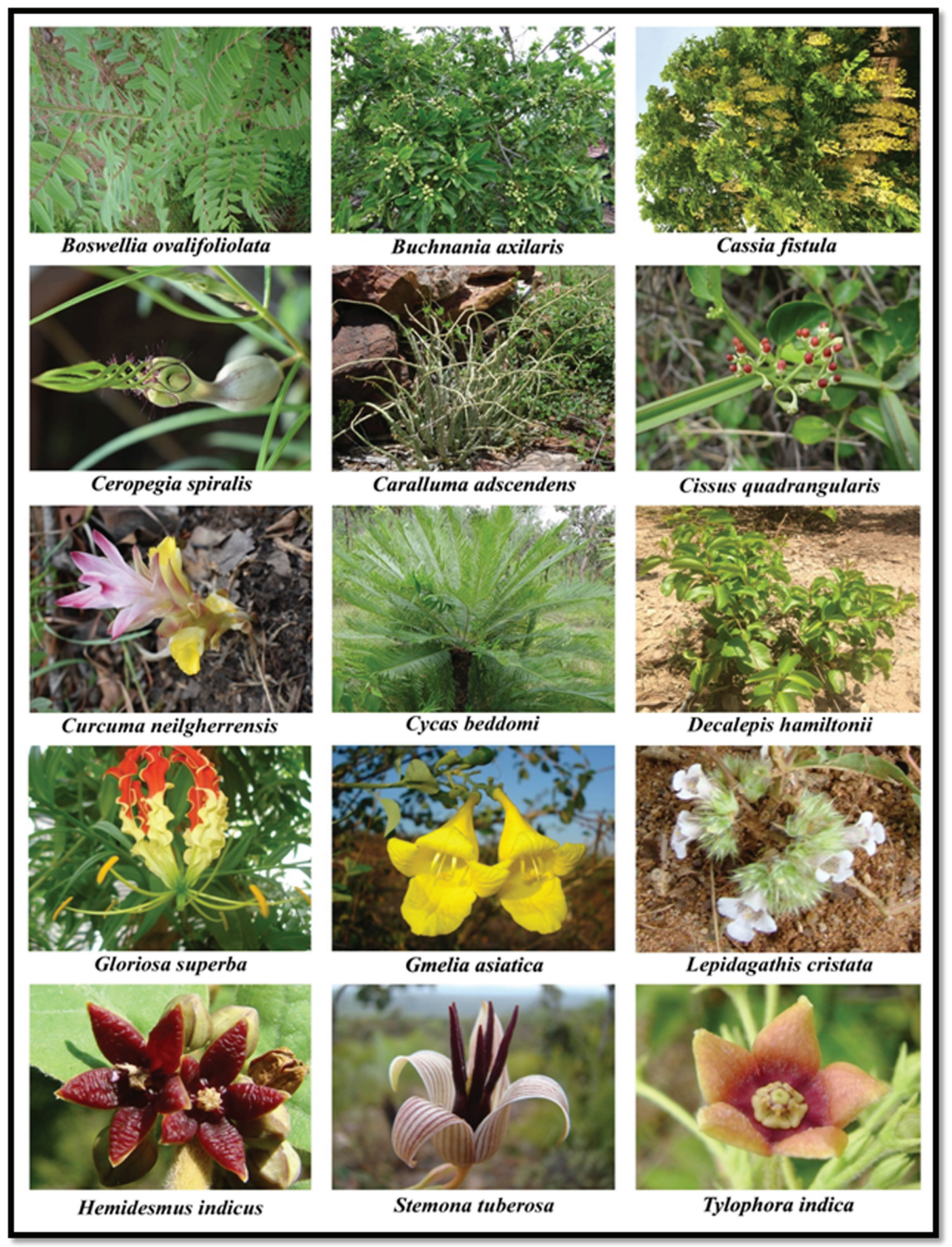

Figure 2

Medicinal Plants of Seshachalam Biosphere Reserve 


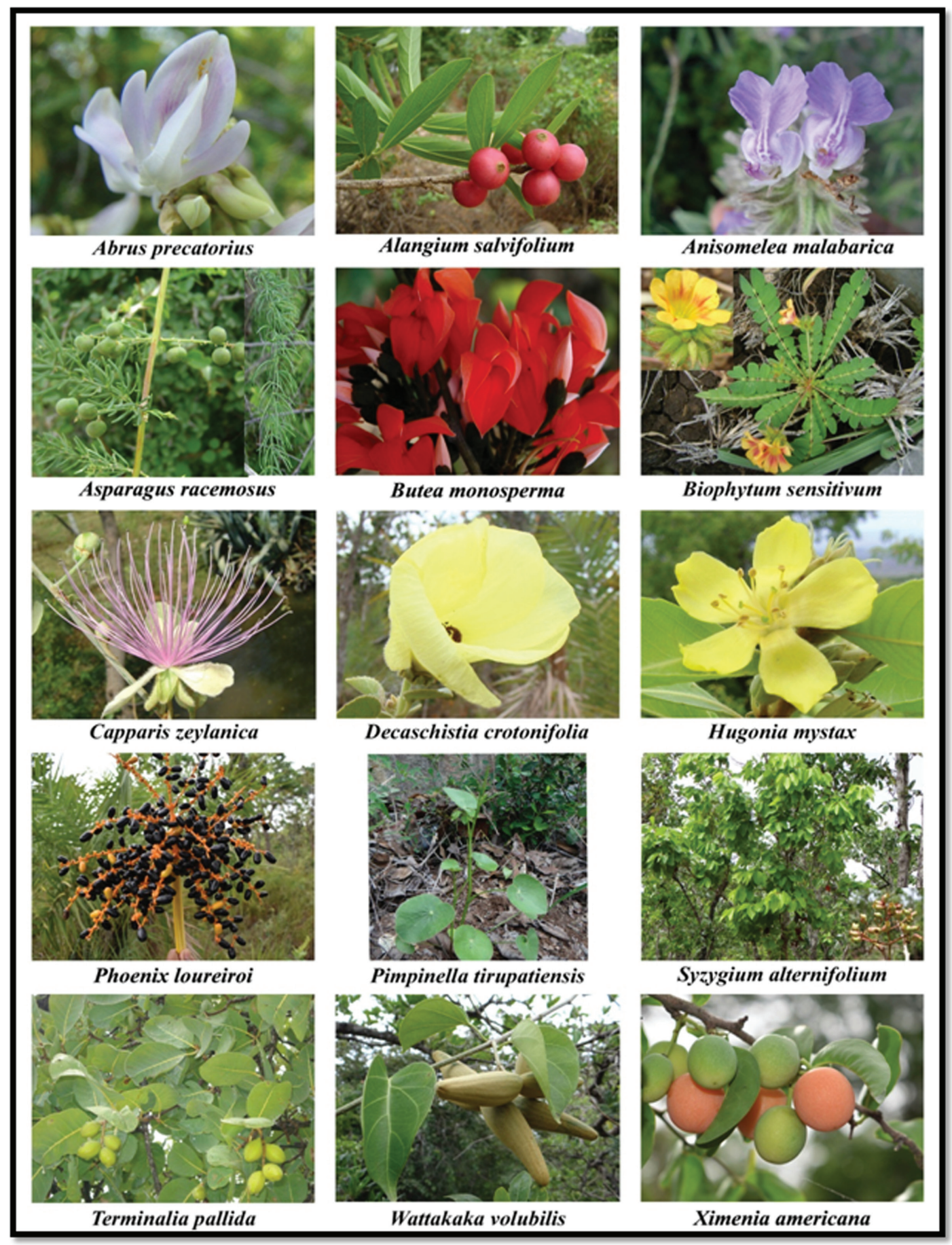

Figure 3

Medicinal Plants of Seshachalam Biosphere Reserve

these are in Red Data Book of Indian Plants. Fascinatingly, in the present study 56 endemic species of southern India are found and are used as traditional herbal plants [6].

The uses of therapeutic plants as remedies for treatment of 64 types of ailments are observed in the study area. The main diseases in the present study area are as follows: rheumatism, earache, dyspepsia, jaundice, paralysis, boils, cough, scorpion sting, skin diseases, snake bite, diabetes, dysentery, gastric troubles, fever, foot cracks, general debility, gout, insects ting, mouth ulcers, stomach-ache, tooth-ache and wounds. The most of medicinal plants are used for the treatment of indigestion, snake bite and skin diseases. The mainstream of medicines has been taken orally and in external application (applied topically on skin). To recover the suitability of certain oral remedies, additives 
are frequently used. The herbal preparation in the area are drawn from a single plant, mixtures may be used rarely. The plant parts used in the study area are leaves, root, bark and whole plant.

\section{CONCLUSIONS}

The medico-botanical investigation of the Seshalam Biosphere area has revealed that the tribes possess good knowledge on plant-based medicine but as the people are towards in advanced exposure to transformation, their information on traditional uses of plants is slowly getting eroded. Therefore, it is significant to study and record the uses of plants by different tribes and sub-tribes on priority aimed at future investigation for product development. These studies may also afford some clues to natural products scientists for phytochemical analysis for any novel molecules or chemicals for treating various ailments.

\section{ACKNOWLEDGEMENT}

The authors are thankful to the Ministry of Environment Forests and Climate Change (MoEF \& CC) (No. 10/16/2016-CS/BG, dated: 31-03-2017) for financial support and also thank to forest officials of Chittoor and Kadapa district for their cooperation in exploration of medicinal plants.

Conflict of interest: Authors declare no conflict of interest.

\section{REFERENCES}

1. Sen S, Chakraborty R. Toward the integration and advancement of herbal medicine: a focus on Traditional Indian medicine. Bot Target Ther 2015; 5:33-44.

2. Hemadri K, Sarma CRR, Rao SS. Medicinal plants wealth of Andhra Pradesh. Part 1. Ancient Sci Life 1987; 6:167-168.

3. Hemadri K, Sarma CRR, Rao SS. Medicinal plants wealth of Andhra Pradesh. Part 2. Ancient
Sci Life 1987; 7:55-64.

4. Vedavathy S, Rao KN, Rajaiah M, Nagaraju N. Folklore information from Rayalaseema region, Andhra Pradesh for family planning and birth control. Int J Pharmacognosy 1991; 29:113-116.

5. Vedavathy S. Herbal folk medicine of Yanadis of Andhra Pradesh. Ethnobotany 1996; 8:109-111.

6. Sudarsanam G, Siva P. Medicinal ethnobotany of plants used as antidotes by Yanadi tribes in south India. J Herbs Spices Med Pl 1995; 3(1):57-66.

7. Sudhakar A, Madhava Chetty K. Medicinal importance of some angiospermic weeds used by the rural people of Chittoor district of Andhra Pradesh, India. Fitoterapia 1998; LXXIX-B:17-12.

8. Reddy KN, Reddy CS, Trimurthulu G. Ethnobotanical uses for respiratory disorders in Eastern Ghats. Ethnobotan Leaflets 2006; 10:139-148.

9. Champion HG, Seth SK. A revised survey of the forest types of India. Government of India, New Delhi 1968.

10. Shah NC, Joshi MC. An ethnobotanical study of the Kumaon region of India. Econ Bot 1971; 25:414-425.

11. Jain SK. Glimpses of Indian Ethnobotany. Oxford IBH Publishing Company, New Delhi 1981:1-334.

12. Shah NC. Main centers and workers of ethnobotany. In: A manual of ethnobotany. Jain SK Scientific Publishers, Jodhpur 1987:69-78.

13. Jain SK, Rao RR. A handbook of field and herbarium methods. Today and Tomorrow's Printers and Publishers, New Delhi 1977:1-157.

14. Gamble JS, Fischer CEC. Flora of the Presidency of Madras. London (Rep. Ed. 1957. BSI, Calcuta) 1915-1935.

15. Pullaiah T et. al. Flora of Andhra Pradesh (vol. 1-5). Scientific Publishers, Jhodpur 2018. 